\title{
Cardiac Sympathetic Dysfunction in Genotyped Patients with Arrhythmogenic Right Ventricular Cardiomyopathy and Risk of Recurrent Ventricular Tachyarrhythmias
}

\author{
Matthias Paul ${ }^{1,2}$, Thomas Wichter ${ }^{1}$, Peter Kies ${ }^{3}$, Joachim Gerss ${ }^{4}$, Christian Wollmann ${ }^{1}$, Kambiz Rahbar $^{3}$, Lars Eckardt ${ }^{1}$, \\ Günter Breithardt ${ }^{1}$, Otmar Schober ${ }^{3}$, Eric Schulze-Bahr ${ }^{1,2}$, and Michael Schäfers ${ }^{3,5}$ \\ ${ }^{1}$ Department of Cardiology and Angiology, University Hospital Münster, Münster, Germany; ${ }^{2}$ Institute for Genetics of Heart Diseases, \\ University Hospital Münster, Münster, Germany; ${ }^{3}$ Department of Nuclear Medicine, University Hospital Münster, Münster, Germany; \\ ${ }^{4}$ Institute of Biostatistics and Clinical Research, University of Münster, Münster, Germany; and ${ }^{5}$ European Institute for Molecular \\ Imaging (EIMI), University of Münster, Münster, Germany
}

Patients with arrhythmogenic right ventricular cardiomyopathy (ARVC) typically present with ventricular tachyarrhythmias preferentially triggered by an elevated sympathetic tone. Previous studies demonstrated an impairment of the presynaptic catecholamine reuptake as assessed by ${ }^{123}$-labeled norepinephrine analog on metaiodobenzylguanidine (123/-MIBG) SPECT. Mutations in the gene encoding for plakophilin-2 (PKP-2) are the most common cause of autosomal dominant ARVC (ARVC-9). In this study, we investigated the potential role of adrenergic dysfunction on the arrhythmia profile in patients with ARVC and correlated these findings with the causative genotype. Methods: ${ }^{123}$ I-MIBG SPECT was performed for 42 patients with definite ARVC (10 women, 32 men; mean age $\pm \mathrm{SD}, 43 \pm 14 \mathrm{y})$. Images were acquired at $4 \mathrm{~h}$ after injection and analyzed for regional ${ }^{123}$ I-MIBG uptake in a standardized 33segment polar map. Results were compared with those obtained from 10 control subjects ( 5 women, 5 men; mean age \pm SD, $43 \pm 12$ y). Results: An abnormal tracer uptake was detected in 25 patients with ARVC (59\%). The extents of right ventricular dilation and regional wall motion abnormalities as well as electrocardiographic markers of de- or repolarization were not significantly different between patients with normal and abnormal 123|-MIBG SPECT findings. However, during long-term follow-up of $11.9 \pm 4.1 \mathrm{y}$, patients with abnormal ${ }^{123}$ |-MIBG SPECT findings experienced life-threatening ventricular tachyarrhythmias significantly more often (22/25 patients [88\%]) and independent of the extent of right ventricular dysfunction than those with a normal sympathetic innervation (6/17 patients [35\%]; $P<0.0005)$. Mutations in $P K P-2$ were identified in 17 patients (40\%) but were not correlated with the degree of adrenergic dysfunction. Conclusion: In patients with ARVC, an impairment of adrenergic innervation independent of the underlying genotype is associated with a higher incidence for future recurrences of ventricular tachyarrhythmias. This finding may suggest a potential role of ${ }^{123}$ I-MIBG SPECT for individualized risk stratification in ARVC patients and asymptomatic $P K P-2$ mutation carriers alike.

\footnotetext{
Received Feb. 7, 2010; revision accepted Jul. 29, 2011.

For correspondence or reprints contact: Matthias Paul, Department für Kardiologie und Angiologie, Universitätsklinikum Münster, Albert-SchweitzerCampus 1, D-48149 Münster, Germany.

E-mail: Matthias.Paul@ukmuenster.de

Published online Sep. 9, 2011.

COPYRIGHT ( 2011 by the Society of Nuclear Medicine, Inc.
}

Key Words: arrhythmogenic right ventricular cardiomyopathy; scintigraphy; ${ }^{123}$ I-MIBG; sympathetic nervous system; risk stratification

J Nucl Med 2011; 52:1559-1565

DOI: 10.2967/jnumed.111.088997

$\mathbf{I}$ dentification of new and refinement of existing methods of risk stratification to prevent sudden cardiac arrest is essential. Recent data from a large multicenter trial in heart failure patients highlighted the independent prognostic value of ${ }^{123}$ I-labeled norepinephrine analog on metaiodobenzylguanidine ( ${ }^{123} \mathrm{I}-\mathrm{MIBG}$; Covidien) SPECT in assessing the risk of future ventricular tachyarrhythmias (1).

Our group was the first, to our knowledge, to report on regional abnormalities of the presynaptic myocardial sympathetic function using ${ }^{123}$ I-MIBG SPECT in primarily non-heart-failure patients with arrhythmogenic right ventricular cardiomyopathy (ARVC) (2). This disease is one of the major causes of sudden death in the young. Characteristic localized or diffuse replacement of predominantly right ventricular (RV) myocardium by fibrous fatty tissue gives rise to global and regional RV (and occasionally left ventricular [LV]) dysfunction and causes a predisposition to life-threatening ventricular tachyarrhythmias (3). Pedigree analyses suggested a Mendelian pattern of inheritance, and the majority of mutations identified so far afflict desmosomal proteins as the key architectural components of heart muscle cell integrity, most of them located in the gene encoding for plakophilin-2 (PKP-2) (4). To date, the role of ${ }^{123}$ I-MIBG SPECT and its potential implication for the arrhythmia profile among other methods of risk stratification (5) in ARVC patients still remain to be elucidated. Therefore, we prospectively evaluated the sympathetic function by ${ }^{123}$ I-MIBG SPECT, correlated the results with the long-term course of the disease in genotyped patients with ARVC (ARVC-9), and additionally evaluated the role of the genotype in this context. 


\section{MATERIALS AND METHODS}

\section{Study Patients, Methods, and Control Group}

Between March 1992 and July 2003, 42 patients with definite ARVC underwent ${ }^{123}$ I-MIBG SPECT (10 women, 32 men; mean age \pm SD at SPECT scan, $43 \pm 14$ y). Diagnosis of ARVC was based on detailed noninvasive investigations (12-lead electrocardiogram and transthoracic echocardiography in all patients [100\%]; MRI before ${ }^{123}$ I-MIBG SPECT in 17 patients [40\%; Table 1]) and invasive investigations (right and left ventriculography and programmed ventricular stimulation as reported earlier (2) in all patients [100\%; Table 1]), in accordance with established and recently modified diagnostic criteria (6). No patient showed clinical signs or symptoms of ischemia; all had normal exercise stress tests as well as normal coronary angiograms.

$\mathrm{RV}$ angiography in a biplane view (i.e., $30^{\circ}$ right anterior oblique projection; $60^{\circ}$ left anterior oblique projection) was used for evaluation of RV size and wall motion (Table 1). RV angiograms were visually evaluated in consensus by 2 experienced cardiologists unaware of the ${ }^{123}$ I-MIBG SPECT findings as to the extent of global RV dilatation and regional RV dysfunction (absent, moderate, or severe).

The initial arrhythmia manifestation leading to the diagnosis of ARVC encompassed nonsustained ventricular tachycardia (VT; i.e., lasting $\geq 10$ consecutive QRS complexes with spontaneous termination within $30 \mathrm{~s}$ ), sustained VT (i.e., lasting $>30$ s or requiring termination due to hemodynamic deterioration), and ventricular fibrillation (VF). The clinical characteristics are summarized in Table 1.
Complete long-term follow-up data were available in all patients. Follow-up started with the date of ${ }^{123}$ I-MIBG SPECT and ended at the last follow-up visit, heart transplantation, or death (allocated by cause: cardiac vs. noncardiac; death validations were based on the hospital records of the patients' last attended hospital; Table 2). During follow-up, the first occurrence of any sustained VT or VF served as the primary clinical endpoint. Follow-up data were obtained during scheduled visits in our outpatient clinic, during regular telephone calls with patients or referring physicians at regular intervals, and when an adverse event occurred (e.g., VT occurrence). In those patients with an implanted cardioverter defibrillator (ICD), interrogations of the device were scheduled every 3 mo. An ICD therapy (antitachycardic pacing or shock) was classified as appropriate if the stored electrograms or RR intervals retrieved from the ICD confirmed that the tachyarrhythmia before the first treatment by the device was sustained and of ventricular origin.

Molecular genetic analyses from venous blood samples of the ARVC patients and from 380 unrelated controls were performed to detect mutations in the $P K P-2$ gene according to the protocol published earlier (4). All coding regions and intron boundaries of the $P K P-2$ gene were directly sequenced and compared with reference sequences.

\section{Control Group}

Ten patients ( 5 men, 5 women; mean age $\pm \mathrm{SD}, 43 \pm 12 \mathrm{y}$; $P=$ not significant vs. study group) with medullary carcinoma of

TABLE 1

Clinical Characteristics of Study Patients

\begin{tabular}{|c|c|c|c|c|c|}
\hline \multirow[b]{2}{*}{ Characteristic } & \multicolumn{3}{|c|}{ ARVC } & \multirow[b]{2}{*}{ Controls } & \multirow[b]{2}{*}{$P$} \\
\hline & All & $\mathrm{MIBG}_{\mathrm{abnormal}}$ & $\mathrm{MIBG}_{\text {normal }}$ & & \\
\hline Patient age at ${ }^{123 \mid-M I B G}$ scan (y) & $43 \pm 14$ & $46 \pm 14$ & $38 \pm 14$ & $43 \pm 12$ & 0.211 \\
\hline Patient age at initial diagnosis $(\mathrm{y})$ & $40 \pm 13$ & $43 \pm 14$ & $36 \pm 13$ & - & 0.106 \\
\hline $\begin{array}{l}\text { Time interval between initial } \\
\text { VT }{ }^{123} \text { |-MIBG scan }(\mathrm{y})\end{array}$ & $5.0 \pm 7.1$ & $5.8 \pm 8.2$ & $3.9 \pm 5.1$ & - & 0.419 \\
\hline Patient age at last follow-up (y) & $55 \pm 15$ & $57 \pm 15$ & $51 \pm 15$ & - & 0.244 \\
\hline Sex & & & & & 0.482 \\
\hline Male & 32 & 20 & 12 & 5 & \\
\hline Female & 10 & 5 & 5 & 5 & \\
\hline Family history $(n)$ & $8(19)$ & $4(16)$ & $4(24)$ & - & 0.542 \\
\hline MRI available before ${ }^{123}$ I-MIBG scan $(n)$ & $17(40)$ & $9(36)$ & $8(47)$ & - & 0.851 \\
\hline \multicolumn{6}{|l|}{ Structural abnormalities (RV angiogram) } \\
\hline \multicolumn{6}{|l|}{ RV size } \\
\hline No RV dilation $(n)$ & $3(7)$ & $1(4)$ & $2(12)$ & - & \\
\hline Moderate RV-dilation ( $n$ ) & $27(64)$ & $16(64)$ & $11(65)$ & - & 0.574 \\
\hline Severe RV-dilation $(n)$ & $12(29)$ & $8(32)$ & $4(23)$ & - & \\
\hline \multicolumn{6}{|l|}{ RV wall motion } \\
\hline No abnormalities $(n)$ & 0 & 0 & 0 & - & \\
\hline Moderate abnormalities $(n)$ & $18(43)$ & $9(36)$ & $9(53)$ & - & 0.276 \\
\hline Severe abnormalities $(n)$ & $24(57)$ & $16(64)$ & $8(47)$ & - & \\
\hline LV involvement (LV angiogram) (n) & $10(25)$ & $8(32)$ & $2(12)$ & - & 0.131 \\
\hline \multicolumn{6}{|l|}{ Electrocardiogram characteristics } \\
\hline Inverted T-waves $(n)$ & $2.5 \pm 1.8$ & $2.5 \pm 1.8$ & $2.6 \pm 1.9$ & - & 0.773 \\
\hline Maximal QRS duration (ms) & $106 \pm 18$ & $108 \pm 21$ & $103 \pm 14$ & - & 0.369 \\
\hline$\epsilon$-potential $(n)$ & $6(14)$ & $5(20)$ & $1(6)$ & - & 0.199 \\
\hline \multicolumn{6}{|l|}{ Genetics } \\
\hline Presence of a $P K P-2$ mutation $(n)$ & $17(40)$ & $11(44)$ & $6(35)$ & - & 0.573 \\
\hline
\end{tabular}

Unless otherwise indicated, data are mean $\pm \mathrm{SD}$, and data in parentheses are percentages. 
TABLE 2

Arrhythmia Profile of Study Patients

\begin{tabular}{|c|c|c|c|c|}
\hline \multirow[b]{2}{*}{ Parameter } & \multicolumn{3}{|c|}{ ARVC } & \multirow[b]{2}{*}{$P$} \\
\hline & All & $\mathrm{MIBG}_{\mathrm{abnormal}}$ & $\mathrm{MIBG}_{\text {normal }}$ & \\
\hline \multicolumn{5}{|l|}{ Arrhythmias before initial diagnosis } \\
\hline Syncope $(n)$ & $1(2)$ & $1(4)$ & 0 & \\
\hline Nonsustained VT (n) & $9(21)$ & $3(12)$ & $6(35)$ & \\
\hline Cycle length of nonsustained VT (ms) & $308 \pm 54$ & $360 \pm 14$ & $282 \pm 46$ & 0.155 \\
\hline Sustained VT $(n)$ & $25(59)$ & $15(60)$ & $10(59)$ & \\
\hline Cycle length of sustained VT (ms) & $294 \pm 56$ & $294 \pm 49$ & $295 \pm 69$ & \\
\hline $\operatorname{VF}(n)$ & $7(17)$ & $6(24)$ & $1(6)$ & \\
\hline \multicolumn{5}{|l|}{ Programmed ventricular stimulation } \\
\hline Inducible sustained VT $(n)$ & $30(71)$ & $21(84)$ & $9(53)$ & 0.029 \\
\hline Cycle length of induced VT (ms) & $309 \pm 107$ & $330 \pm 117$ & $254 \pm 43$ & 0.089 \\
\hline \multicolumn{5}{|l|}{ Implantable cardioverter defibrillator } \\
\hline Patients with ICD $(n)$ & $23(52)$ & $17(68)$ & $6(35)$ & 0.037 \\
\hline Age at first implantation $(\mathrm{y})$ & $44 \pm 17$ & $48 \pm 17$ & $34 \pm 18$ & 0.098 \\
\hline \multicolumn{5}{|l|}{ Follow-up (after ${ }^{123}$ I-MIBG SPECT) } \\
\hline Duration of follow-up $(y)$ & $11.9 \pm 4.1$ & $11.1 \pm 4.4$ & $13.1 \pm 3.5$ & 0.111 \\
\hline Patients alive $(n)$ & $38(91)$ & $21(84)$ & $17(100)$ & 0.083 \\
\hline Cardiac death $(n)$ & $2(5)$ & $2(8)$ & 0 & \\
\hline Heart transplantation during follow-up (n) & $2(8)$ & $2(8)$ & 0 & \\
\hline Patients with VT/VF during follow-up $(n)$ & $28(67)$ & $22(88)$ & $6(35)$ & $<0.0005$ \\
\hline Time to first VT/VF occurrence $(\mathrm{y})$ & $1.9 \pm 2.7$ & $1.5 \pm 2.5$ & $3.4 \pm 3.1$ & 0.122 \\
\hline \multicolumn{5}{|l|}{ Classification of arrhythmias } \\
\hline Nonsustained VT $(n)$ & $7(25)$ & $5(23)$ & $2(33)$ & \\
\hline Sustained VT (n) & $20(71)$ & $16(73)$ & $4(67)$ & \\
\hline VF $(n)$ & $1(4)$ & $1(4)$ & 0 & \\
\hline Cycle length of nonsustained VT during follow-up (ms) & $300 \pm 72$ & $321 \pm 33$ & $272 \pm 110$ & 0.426 \\
\hline Cycle length of sustained VT during follow-up (ms) & $298 \pm 66$ & $295 \pm 72$ & $310 \pm 35$ & 0.732 \\
\hline Time to first occurrence (y) & $1.9 \pm 2.7$ & $1.5 \pm 2.5$ & $3.4 \pm 3.1$ & 0.122 \\
\hline
\end{tabular}

Unless otherwise indicated, data are mean $\pm \mathrm{SD}$, and data in parentheses are percentages.

the thyroid gland served as an age-matched control group (2). These patients underwent ${ }^{123}$ I-MIBG SPECT to exclude pheochromocytoma. Results of cardiovascular examination, resting 12-lead electrocardiogram, and exercise tests were normal in all patients. No subject of this group was on drug treatment or had a history, sign, or symptom of cardiac disease or of diseases possibly affecting the cardiac autonomic nervous system.

This study was conducted with the approval of the Ethics Committee of the Chamber of Physicians Westfalen-Lippe and the Medical Faculty of the Westphalian Wilhelms-University Münster, Germany, after informed consent was obtained from the patients.

\section{Data Acquisition and Data Analysis}

Cardiac presynaptic sympathetic innervation was assessed by ${ }^{123}$ I-MIBG SPECT in both controls and ARVC patients. No patient had diabetes mellitus or renal or liver disease, which might influence ${ }^{123}$ I-MIBG uptake or metabolism.

Data acquisition and processing were performed according to a protocol published earlier $(2,7,8)$. In brief, after blockage of the thyroid gland with $300 \mathrm{mg}$ of perchlorate, a dose of $350 \mathrm{MBq}$ of commercially available ${ }^{123}$ I-MIBG was administered intravenously, and SPECT images were acquired at $4 \mathrm{~h}$ after injection. For quantitative analyses, the regional ${ }^{123}$ I-MIBG uptake in the LV myocardium was measured using a 33-segment polar map analysis as the percentage uptake relative to the segment with maximal uptake $(100 \%)$. A reduction of tracer uptake in at least 3 adjacent segments by more than 2 SDs of the mean value of the same segment in the control group was defined as an abnormal reduction of ${ }^{123}$ I-MIBG uptake. By calculating the total area of abnormal segments of the polar map, the extent of the affected LV myocardium was assessed. Myocardial ${ }^{201} \mathrm{Tl}$ stress-redistribution SPECT, as a parameter for perfusion and viability, was additionally performed in a subgroup of 8 patients to exclude myocardial abnormalities on a small vessel level in the presence of normal coronary angiograms. All patients demonstrated normal ${ }^{201} \mathrm{Tl}$ SPECT perfusion imaging, despite regional defects in ${ }^{123} \mathrm{I}-\mathrm{MIBG}$ uptake, thus excluding impaired perfusion as a cause of abnormal ${ }^{123}$ I-MIBG results. To minimize radiation exposure for the residual study population, we decided not to perform ${ }^{201} \mathrm{Tl}$ SPECT in the entire cohort.

\section{Statistics}

Statistical analyses were performed using descriptive and inductive statistical methods. Differences of binary target variables between patients and control subjects were assessed using the $\chi^{2}$ test. $P$ values of less than 0.05 were regarded, in general, to be significant. To identify differences in regional ${ }^{123}$ I-MIBG uptake, the $\chi^{2}$ test with Bonferroni adjustment for multiple testing was used. In this case, $P$ values of less than 0.002 were considered significant and a $P$ of less than 0.05 as borderline significant. Metric data are expressed as mean \pm SD. In addition, $z$ scores ([segmental value controls - segmental value patient]/SD controls) 
were calculated where applicable, to evaluate the depth of the innervation defect (Fig. 1). Differences of metric target variables between groups were assessed by parametric ANOVA or Student $t$ test as appropriate. To account for variable lengths of follow-up, the probability of remaining arrhythmia/event-free survival was analyzed by the Kaplan-Meier method, and differences in eventfree survival between groups were evaluated by the log rank test. Additionally multivariate Cox regression analysis was performed. $P$ values of less than 0.05 were considered statistically significant (SPSS, version 16.0 [SPSS Inc.] for Windows [Microsoft]).

\section{RESULTS}

\section{3|-MIBG SPECT in ARVC Patients}

In the present series of 42 patients with ARVC, 358 (26\%) of 1,386 polar map segments showed a significant reduction of LV ${ }^{123}$ I-MIBG uptake. On the aforementioned criteria, SPECT images were normal in 17 patients $\left(\mathrm{MIBG}_{\text {normal }}\right.$; $41 \%)$ and classified as abnormal in 25 patients $\left(\mathrm{MIBG}_{\text {abnormal }}\right.$; $59 \%$ ), with a predominantly reduced tracer uptake either in the septal (32\%), inferior (31\%), or anterior (29\%) segments (Fig. 1; Supplemental Table 1 [supplemental materials are available online only at http://jnm.snmjournals.org]).

\section{Clinical Characteristics and Follow-up in ARVC Patients in Relation to ${ }^{123}$-MIBG SPECT}

The mean time interval between the first ventricular tachyarrhythmia index event and study entry (i.e., date of ${ }^{123}$ I-MIBG SPECT) was $5.0 \pm 7.1 \mathrm{y}$. In 14 of 42 patients $(33 \%)$, sustained ( $n=9$ patients) and nonsustained $(n=5$ patients) VT occurred between initial diagnosis and ${ }^{123} \mathrm{I}-$ MIBG SPECT, which in arrhythmia type and frequency was not correlated with an abnormal ${ }^{123}$ I-MIBG uptake in the images acquired later $(P=0.666)$. In the 1 patient with prior syncope, 3 of 9 patients with nonsustained VT, and 21 of 25 patients with sustained VT as initial clinical arrhythmia, sustained VTs were inducible during programmed ventricular stimulation $(P<0.007)$. An ICD was implanted in 23 of the 42 ARVC patients (55\%): 7 patients $(30 \%)$ received an ICD after a survived episode of sudden cardiac arrest; 16 patients $(70 \%)$ with prior sustained $(n=14)$ or nonsustained VT $(n=2)$ in the presence of an advanced disease manifestation also received an ICD. Baseline clinical characteristics and the length of the follow-up period were not significantly different between the groups (Table 1). However, compared with patients with a normal ${ }^{123} \mathrm{I}-$ MIBG SPECT finding, in MIBG abnormal $_{\text {patients, sustained }}$ VTs were more frequently induced during programmed ventricular stimulation $(84 \%$ vs. $53 \% ; P=0.029$; Table 2 ), and ICDs were more frequently implanted (68\% vs. $35 \% ; P=0.037$; Table 2).

During long-term follow-up of $11.9 \pm 4.1 \mathrm{y}, 28$ patients (67\%) experienced ventricular tachyarrhythmias, which in most cases were classified as sustained VT (71\%; Table 2). In patients with ARVC, the mean time to the first VT or VF occurrence was $1.9 \pm 2.7$ y and did not differ between $\mathrm{MIBG}_{\mathrm{abnormal}}$ and $\mathrm{MIBG}_{\text {normal }}$ patients (Table 2). However, $\mathrm{MIBG}_{\mathrm{abnormal}}$ patients experienced ventricular tachyarrhythmias more often than did $\mathrm{MIBG}_{\text {normal }}$ patients $(88 \%$ vs. $35 \% ; P<0.0005$; Table 2 ), resulting in a significantly worse event-free survival in the Kaplan-Meier analyses $(P$ $<0.001$; Fig. 2). Multivariate Cox regression analysis was performed including the covariates RV size, LV involvement, the presence of an ICD, and an abnormal ${ }^{123}$ I-MIBG SPECT finding. RV size, ICD, and abnormal ${ }^{123} \mathrm{I}-\mathrm{MIBG}$ SPECT finding yet remained independent predictors of a future VT (abnormal ${ }^{123}$ I-MIBG SPECT: hazard ratio, 4.67; 95\% confidence interval, 1.58-13.74; $P=0.005$; Table 3 ).

Four patients (9\%) died during a mean follow-up of $11.9 \pm$ $4.1 \mathrm{y}, 2$ of them (both $\mathrm{MIBG}_{\text {abnormal }}$; Table 2) from progressive biventricular heart failure 14.4 and $14.0 \mathrm{y}$ after the initial diagnosis of ARVC. The other 2 patients died in the wake of carcinoma of the gallbladder and pneumonia. In all cases, the families refused autopsies. Another 2 patients (both $\mathrm{MIBG}_{\mathrm{ab}}$ normal) underwent heart transplantation due to intractable heart failure $5.3 \pm 1.7 \mathrm{y}$ after ${ }^{123} \mathrm{I}$-MIBG SPECT.

Molecular genetic analyses identified mutations in PKP2 in 17 ARVC patients (40\%). The number of mutation

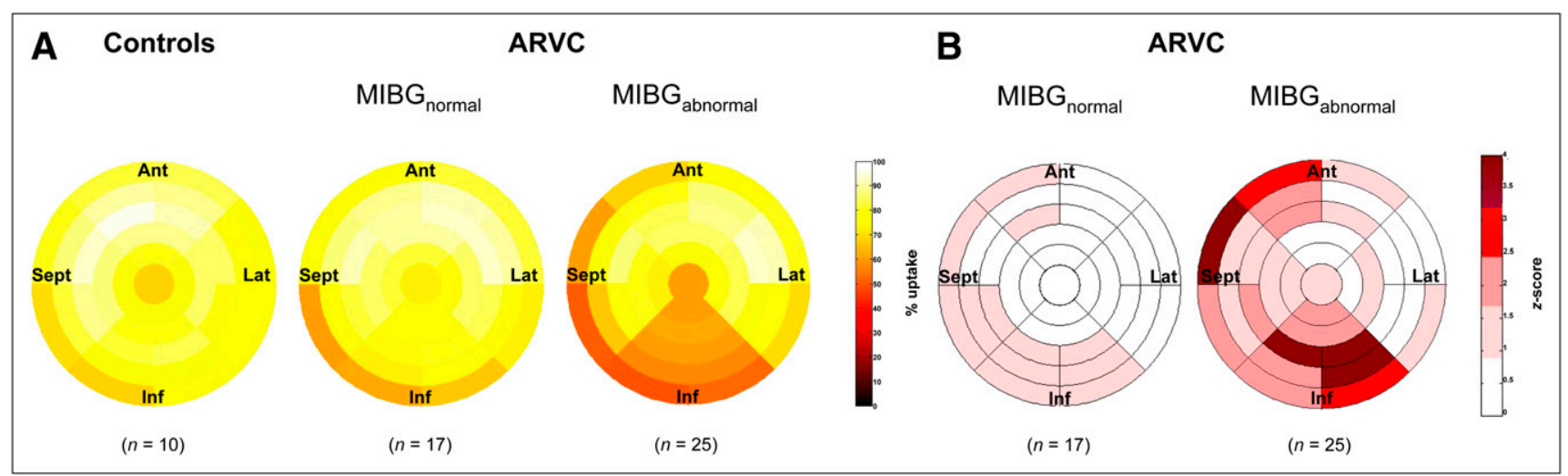

FIGURE 1. (A) Mean segmental ${ }^{123}$ I-MIBG uptake (\%) in ARVC patients and controls. Differences between patients with normal (MIBG $\mathrm{normal}$ and reduced (MIBG abnormal ${ }^{123}$-MIBG uptake in relation to control group (B) are expressed in $z$ scores. Ant = anterior wall segment; Inf $=$ inferior wall segment; Lat $=$ lateral wall segment; Sept $=$ septal wall segment. 


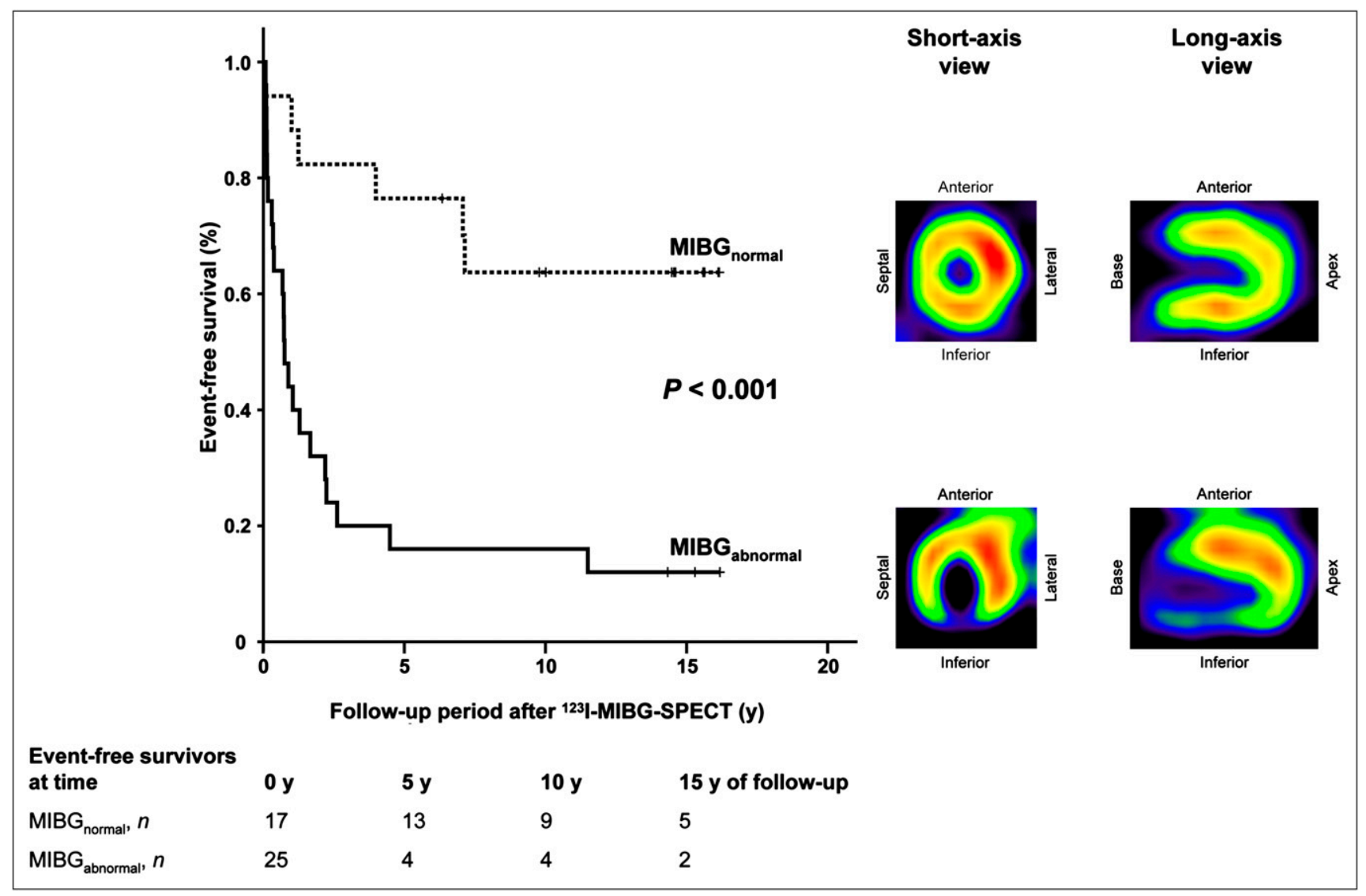

FIGURE 2. Event-free survival (i.e., sustained VT) during years of follow-up of study population with normal (MIBG $\mathrm{normal})$ vs. abnormal $\left(\mathrm{MIBG}_{\text {abnormal }}\right)^{123}$ I-MIBG uptake. At right are typical ${ }^{123}$ I-MIBG images.

carriers among patients with abnormal ${ }^{123}$ I-MIBG SPECT findings was 11 of $25(44 \%)$ and comparable with the 6 mutation carriers among 17 patients $(35 \%)$ with normal ${ }^{123}$ I-MIBG SPECT findings $(P=0.573$; Table 1$)$. During long-term follow-up, Kaplan-Meier analysis of the eventfree survival was statistically not significant between MIB$\mathrm{G}_{\mathrm{abnormal}}$ patients with or without a PKP-2 gene mutation.

\section{DISCUSSION}

There is growing evidence that adrenergic dysfunction assessable by ${ }^{123}$ I-MIBG SPECT indicates a worse prognosis in patients with heart failure (1). In the present study, we could confirm and also expand this finding to the nonheart-failure group of patients with ARVC. The results highlight that ARVC patients with an abnormal ${ }^{123}$ I-MIBG uptake have a significantly higher propensity for life-threatening ventricular tachyarrhythmias than those with a normal ${ }^{123}$ I-MIBG SPECT result during long-term follow-up. This finding was independent of the initial extent of the underlying RV dysfunction, and a PKP-2 genotype did not add to the prognostic value.

Given that ${ }^{123}$ I-MIBG uptake was assessed at $4 \mathrm{~h}$ after injection, no conclusions on the dynamics of ${ }^{123}$ I-MIBG uptake and release can be drawn. However, the described reduction of tracer activity in the inferior parts of the left ventricle might result from either a reduced reuptake (uptake-1) of ${ }^{123}$ I-MIBG or catecholamines, an increased release of ${ }^{123} \mathrm{I}-\mathrm{MIBG}$ or catecholamines into the synaptic cleft, or a combination of both. All mechanisms would eventually lead to an enhanced catecholamine concentration in the synaptic cleft, with subsequent downregulation of postsynaptic $\beta$-adrenoceptor density, as we have previously shown by quantitative PET (9).

There was a significantly reduced ${ }^{123}$ I-MIBG uptake in anterior, septal, and inferior myocardial segments in patients with ARVC $(P<0.001$; Fig. 1). The uptake of ${ }^{123} \mathrm{I}-\mathrm{MIBG}$ is known to be reduced in the inferior wall in the cases of increased parasympathetic tone. This reduction is probably caused by the more pronounced parasympathetic innervation of the inferior LV wall and the RV when compared with the remaining myocardium (10), leading to a mismatch between parasympathetic and sympathetic innervation in the inferior wall and resulting in reduced inferior ${ }^{123} \mathrm{I}-\mathrm{MIBG}$ uptake. Myocardial hypoperfusion as a potentially confounding source leading to sympathetic dysinnervation appears unlikely in the presence of normal coronary angiograms in all patients and normal ${ }^{201} \mathrm{Tl}$ stress-redistribution SPECT findings in a subset of patients. The definite mechanism linking ${ }^{123}$ I-MIBG SPECT results with VT or VF recurrences remains yet to be elucidated. 
TABLE 3

Predictors of VT During Follow-up Identified by Multivariate Cox Regression

\begin{tabular}{lll}
\hline \multicolumn{1}{c}{ Model } & \multicolumn{2}{c}{ ARVC } \\
\cline { 2 - 3 } & Hazard ratio (VT) & $95 \%$ confidence interval \\
\hline Initial & & \\
$\quad$ Structural abnormalities (on angiogram) & & \\
$\quad$ RV size & 0.120 & $0.02-0.74$ \\
Moderate vs. no RV dilation & 0.147 & $0.02-1.01$ \\
Severe vs. no RV dilation & 1.793 & $0.71-4.56$ \\
$\quad$ LV involvement & & \\
Presence of an... & 3.532 & $1.23-10.15$ \\
ICD & 4.037 & $1.35-12.12$ \\
Abnormal 123I-MIBG SPECT finding & & \\
Final & & 0.022 \\
RV size & 0.135 & $0.02-0.79$ \\
Moderate vs. no RV dilation & 0.197 & $0.03-1.24$ \\
Severe vs. no RV dilation & & \\
Presence of an... & 3.012 & $1.08-8.41$ \\
ICD & 4.667 & $1.58-13.74$
\end{tabular}

Initial model includes covariates RV size, LV involvement, ICD, and normal/abnormal ${ }^{123}$-MIBG finding, and final model is after (backward) elimination of nonsignificant covariates.

$\mathrm{ICD}=$ automatic cardioverter defibrillator; VT = ventricular tachycardia.

Interestingly, the patterns of ${ }^{123} \mathrm{I}-\mathrm{MIBG}$ uptake deficiencies (location, size, etc.) were not directly correlated with the resulting type of arrhythmias (nonsustained, sustained VT, etc.) or the frequency of arrhythmias, suggesting a nonlinear relation between a disturbed sympathetic nervous system and the occurrence of ventricular tachyarrhythmias. However, no proof of a causal relationship can be deduced from the present study.

Nevertheless, the observed predisposition to future ventricular tachyarrhythmias in patients with a reduced ${ }^{123}$ I-MIBG uptake could eventually lead to the recognition of ${ }^{123}$ I-MIBG SPECT as an indicator of an increased individual risk of future arrhythmic events. This is in line with previous studies in patients with Brugada syndrome (7) or other structural heart diseases (11) and also in a large European multicenter study (12) in which a reduced tracer uptake as a reflection of an autonomic dysfunction was associated with an increased risk of death. These studies, including ours, clearly suggest an emerging role of ${ }^{123} \mathrm{I}-$ MIBG SPECT for individualized risk stratification; the implantation of an ICD could be discussed in those ARVC patients with a sympathetic dysfunction and no prior indication for this device.

The following are limitations of our study. Because the imaging part of the study was already initiated in the 1990s after the first description of ${ }^{123}$ I-MIBG defects in ARVC worldwide by our group (2), state-of-the-art ${ }^{123}$ I-MIBG imaging refers to the time of initiating the study and not the current status of ${ }^{123} \mathrm{I}-\mathrm{MIBG}$ imaging (i.e., acquisition and 33-segment polar map analysis). To facilitate standardized analyses, we intentionally did not change the technique in the course of time. New studies in our center being currently initiated refer to current standards such as early and late imaging and heart-to-mediastinum ratios.

The decision for implantation of an ICD, which was not part of the study, was based on guidelines existing at the time of diagnosis irrespective of the results of ${ }^{123}$ I-MIBG SPECT and on the patient's choice. Genetic analysis focused on the presence or absence of PKP-2 mutations, which underlie up to $43 \%$ of ARVC patients (5), and was complete in all patients. However, information on other known desmosomal gene mutations was incomplete.

The present study was not designed to confirm or refute a potential causal relation of sympathetic dysfunction and predominantly right ventricular tachyarrhythmias. Standard ${ }^{123}$ I-MIBG SPECT is restricted to the left ventricle, and thus no direct information on the sympathetic innervation of the right ventricle can currently be provided by SPECT techniques. However, the high incidence of abnormal ${ }^{123}$ I-MIBG SPECT not only in ARVC patients but also in patients with known nonstructural LV abnormalities such as RV outflowtract tachycardias (8), or with the Brugada syndrome (7) or long-QT syndrome (13), may underscore the value of ${ }^{123} \mathrm{I}-$ MIBG SPECT.

In addition, this study cannot finally resolve the question of whether a reduced receptor density or a reduction of sympathetic capacity itself results in an innervation defect as made visible by ${ }^{123} \mathrm{I}$-MIBG SPECT. To date, no technical means are available to answer this question.

Over recent years, B-type natriuretic peptides and other biomarkers have gained increasing widespread recognition in clinical medicine facilitated by tests then commercially available (14). However, analysis of cardiac biomarkers was not part of this study, which started as early as 1992. 


\section{CONCLUSION}

Presynaptic adrenergic dysfunction assessed by the regional reduction of myocardial uptake is associated with a markedly higher risk of future recurrent life-threatening ventricular tachyarrhythmias in patients with ARVC. ${ }^{123}$ I-MIBG imaging may therefore help to establish an individualized risk stratification to minimize the propensity for ventricular tachyarrhythmias and sudden death in patients with ARVC.

\section{DISCLOSURE STATEMENT}

The costs of publication of this article were defrayed in part by the payment of page charges. Therefore, and solely to indicate this fact, this article is hereby marked "advertisement" in accordance with 18 USC section 1734.

\section{ACKNOWLEDGMENTS}

We thank Petra Gerdes and Antje Hellige for their excellent technical assistance. This work was supported in part by grants from the Deutsche Forschungsgemeinschaft (DFG), Bonn, Germany; Sonderforschungsbereich 656 (Project C1), from the Fondation Leducq, Paris, France; and the European Commission (QLG-CT-2000-01091), Brussels, Belgium. No other potential conflict of interest relevant to this article was reported.

\section{REFERENCES}

1. Jacobson AF, Senior R, Cerqueira MD, et al. ADMIRE-HF Investigators. Myocardial iodine-123 meta-iodobenzylguanidine imaging and cardiac events in heart failure: results of the prospective ADMIRE-HF (AdreView Myocardial
Imaging for Risk Evaluation in Heart Failure) study. J Am Coll Cardiol. 2010;55:2212-2221.

2. Wichter T, Hindricks G, Lerch H, et al. Regional myocardial sympathetic dysinnervation in arrhythmogenic right ventricular cardiomyopathy: an analysis using ${ }^{123}$ I-meta-iodobenzylguanidine scintigraphy. Circulation. 1994;89:667-683.

3. Marcus FI, Fontaine GH, Guiraudon G, et al. Right ventricular dysplasia: a report of 24 cases. Circulation. 1982;65:384-398.

4. Gerull B, Heuser A, Wichter T, et al. Mutations in the desmosomal protein plakophilin-2 are common in arrhythmogenic right ventricular cardiomyopathy. Nat Genet. 2004;36:1162-1164.

5. Sen-Chowdhry S, Morgan RD, Chambers JC, McKenna WJ. Arrhythmogenic cardiomyopathy: etiology, diagnosis, and treatment. Annu Rev Med. 2010;61:233-253.

6. Marcus FI, McKenna WJ, Sherrill D, et al. Diagnosis of arrhythmogenic right ventricular cardiomyopathy/dysplasia: proposed modification of the task force criteria. Circulation. 2010;121:1533-1541.

7. Wichter T, Matheja P, Eckardt L, et al. Cardiac autonomic dysfunction in Brugada syndrome. Circulation. 2002;105:702-706.

8. Schäfers M, Wichter T, Lerch H, et al. Cardiac ${ }^{123}$ I-MIBG uptake in idiopathic ventricular tachycardia and fibrillation. J Nucl Med. 1999;40:1-5.

9. Wichter T, Schäfers M, Rhodes CG, et al. Abnormalities of cardiac sympathetic innervation in arrhythmogenic right ventricular cardiomyopathy. Quantitative assessment of presynaptic norepinephrine reuptake and postsynaptic $\beta$-adrenergic receptor density with positron emission tomography. Circulation. 2000;101: 1552-1558.

10. Morozumi T, Kusuoka H, Fukuchi K, et al. Myocardial iodine-123-metaiodobenzylguanidine images and autonomic nerve activity in normal subjects. $\mathrm{J}$ Nucl Med. 1997;38:49-52.

11. Nagamatsu H, Momose M, Kobayashi H, Kusakabe K, Kasanuki H. Prognostic value of ${ }^{123} \mathrm{I}$-metaiodobenzylguanidine in patients with various heart diseases. Ann Nucl Med. 2007;21:513-520.

12. Agostini D, Verberne HJ, Burchert W, et al. I-123-mIBG myocardial imaging for assessment of risk for a major cardiac event in heart failure patients: insights from a retrospective European multicenter study. Eur J Nucl Med Mol Imaging. 2008;35:535-546.

13. Göhl K, Feistel H, Weikl A, Bachmann K, Wolf F. Congenital myocardial sympathetic dysinnervation (CMSD)-a structural defect of idiopathic long QT syndrome. Pacing Clin Electrophysiol. 1991;14:1544-1553.

14. O'Donoghue M, Braunwald E. Natriuretic peptides in heart failure: should therapy be guided by BNP levels? Nat Rev Cardiol. 2010;7:13-20. 